\title{
A 68-year-old man with an incidentally discovered pituitary lesion
}

\author{
Omar Saeed MD, Manoela Braga MD
}

Cite as: CMAJ 2017 April 24;189:E605-7. doi: 10.1503/cmaj.151556

A 68-year-old man was initially examined in the emergency department after sustaining trauma to his head after a fall on ice outside his home. He was on therapeutic anticoagulation treatment for atrial fibrillation. A computed tomography (CT) scan of his head did not show any evidence of acute pathology, the examination and additional investigations were reassuring, and he was subsequently discharged home after a period of monitoring. However, the final radiology report sent to his primary care provider indicated the presence of an unexpected sellar mass. He presents to his family physician for follow-up and a discussion regarding this lesion.

\section{What is the likely diagnosis?}

The lesion identified in this case can be classified as a pituitary incidentaloma, which is defined as an anatomic abnormality of the sella identified unexpectedly on an imaging study. ${ }^{1}$ This excludes lesions found during the investigation of symptoms potentially attributable to pituitary disease, such as visual field disturbances, symptoms of hypopituitarism or of pituitary hormone excess. ${ }^{2}$ Pituitary incidentalomas are classified by size as either microincidentalomas (defined as less than $10 \mathrm{~mm}$ in size, representing most incidentally discovered sellar lesions) or macroincidentalomas (10 mm or more in size), a distinction that carries prognostic importance along with implications for management and follow-up. ${ }^{2,3}$

Although the list of potential causes for an incidentally discovered sellar mass is broad, pituitary adenomas are the most common. In a multicentre retrospective study of incidentally discovered sellar masses involving 258 patients who ultimately required surgery, pathologic assessment showed that $81 \%$ had pituitary adenomas, $16 \%$ had Rathke cleft cysts (a cyst formed at Rathke pouch between the anterior and posterior pituitary after failed closure in early fetal development), and the remainder had craniopharyngiomas or arachnoid cysts. ${ }^{4} \mathrm{~A}$ separate unselected autopsy study of 1000 specimens confirmed pituitary adenomas and Rathke cleft cysts as the dominant pathologies for incidentally discovered lesions of the pituitary..$^{5}$

Because most pituitary incidentalomas are adenomas, data on adenomas may provide reliable information on the overall prevalence of incidentally discovered sellar lesions. A systematic review that included 10 studies found a prevalence of $14.4 \%$ for pituitary adenomas based on autopsy studies, $22.5 \%$ based on radiologic studies and $16.7 \%$ across all studies. ${ }^{6}$

\section{What should be included in the initial assessment and physical examination?}

All patients found to have a pituitary incidentaloma should undergo a thorough history and physical examination to identify signs and symptoms of potential pituitary hormone excess or deficiency, and to assess for any visual field deficits due to optic chiasm compression from the lesion. ${ }^{2,3}$

Hormone-secreting lesions usually present with a clinical syndrome reflecting the hormone in excess. For example, prolactinomas, the most common hypersecretory lesions, can cause galactorrhea and hypogonadotropic hypogonadism (e.g., infertility, oligomenorrhea or amenorrhea in women; erectile dysfunction, infertility or gynecomastia in men).

The physical examination should include a detailed assessment of cranial nerves II, III, IV, V (specifically the V1 and V2 distributions) and $\mathrm{VI}$, given the anatomic proximity of the pituitary gland to the optic chiasm and the cavernous sinus. This assessment typically includes testing of visual acuity with a Snellen chart, testing of visual fields by confrontation or a formal visual field test (e.g., to look for bitemporal hemianopsia secondary to midoptic chiasm compression by the pituitary mass), checking for the pupillary light reflex to look for a relative afferent pupillary defect and fundoscopy to look for pallor of the optic disc and, less commonly, papilledema.

Guidelines recommend referral for a formal visual field test for any patient with a lesion in proximity to or showing compression of the optic chiasm or optic nerves based on imaging. ${ }^{2,3}$

\section{Which initial investigations should be ordered?}

Based on the most recently published Endocrine Society ${ }^{2}$ and French Endocrine Society ${ }^{3}$ guidelines for the management of pituitary incidentalomas, a sellar mass found on a CT scan should be further delineated using magnetic resonance imaging (MRI) with a pituitary protocol. This involves fine $(1 \mathrm{~mm})$ slices through the sella, with images obtained before and after contrast enhancement.

Recommendations for the hormonal evaluation of pituitary incidentalomas are heavily based on clinical experience, because literature on this topic is limited. Guidelines advise screening all patients with pituitary incidentalomas for hypopituitarism irrespective of lesion size, because of high reported rates of anterior pituitary hormone deficiency noted in several 
studies. ${ }^{2,7}$ Testing should always be guided by clinical judgment, with the recommended hormonal evaluation for hypopituitarism including serum free thyroxine (free $\mathrm{T}_{4}$ ), thyroid-stimulating hormone (TSH), morning cortisol, adrenocorticotropic hormone (ACTH), luteinizing hormone (LH), follicle-stimulating hormone (FSH), testosterone in men and a detailed menstrual and gonadal function history in women to guide consideration of estradiol assessment. ${ }^{2,3}$

In the context of hormonal hypersecretion, testing for serum prolactin has the strongest evidence because prolactinomas are the most common hypersecretory lesions. ${ }^{8,9}$ Based on the prevalence of previously unsuspected growth hormone-secreting tumours and the association with decreased morbidity after early detection and surgical management, routine screening for acromegaly by measurement of serum insulinlike growth factor 1 (IGF-1) levels is also suggested in the guidelines. ${ }^{2,3}$

Evaluation for cortisol excess (e.g., with a 24-hour urinary free cortisol measurement or a 1-mg overnight dexamethasone suppression test) should be guided by clinical features and not routinely included in the assessment of patients without cushingoid features. ${ }^{2,3}$

\section{How should ongoing follow-up for this lesion be managed?}

Follow-up for pituitary incidentalomas is dependent on the results of the initial clinical assessment, imaging and investigations. The Endocrine Society and French Endocrine Society guidelines also outline criteria for consideration of referral for surgical management (Box 1). ${ }^{2,3}$

Prolactin-secreting tumours are primarily treated medically with dopamine agonists such as cabergoline or bromocriptine. These medications often effectively decrease serum prolactin concentrations and the size of most lactotroph adenomas., ${ }^{8,9}$

Box 1: Potential indications for referral to neurosurgery for a sellar lesion ${ }^{2,3}$

- Visual field deficit attributable to the lesion

- Lesion proximal to or compressing the optic nerves or optic chiasm based on imaging

- Planned pregnancy with a lesion in proximity to the optic chiasm

- Clinically significant hypersecreting lesions other than isolated prolactinomas

- Symptomatic prolactinoma and an inability to tolerate or inadequate response to an appropriate trial of dopamine agonist therapy

- Visual disturbance with pituitary apoplexy

- High risk of apoplexy, such as in patients receiving therapeutic anticoagulation treatment who have macroadenomas proximal to the optic chiasm

- Clinically significant anterior pituitary hormone insufficiency

- Clinically significant growth of the lesion (no threshold for size increase has been established)

- Unremitting headache where a thorough assessment for other causes has been completed
Nonsurgical candidates should be carefully followed. The Endocrine Society guideline for follow-up of macroincidentalomas suggests a repeat MRI of the sella at six months, then annually for three years and less frequently thereafter if the lesion is stable. ${ }^{2}$ For microincidentalomas, follow-up MRI is initially recommended at one year, then every one to two years for the following three years, and less often thereafter if the lesion remains stable. ${ }^{2}$ The French Endocrine Society guideline is in agreement with these recommendations, with the additional recommendation that nonfunctioning pituitary microincidentalomas $5 \mathrm{~mm}$ or less in size do not require repeat imaging, based on studies that showed a low likelihood of progression of these lesions. ${ }^{3}$

In addition, laboratory investigations guided by clinical assessment for hypopituitarism should be repeated at six months and yearly thereafter in patients with macroincidentalomas. Repeat testing for hypopituitarism is unnecessary with microincidentalomas, unless characteristics change substantially on MRI, or if corresponding signs and symptoms develop. ${ }^{2,3}$

\section{Case revisited}

Our patient underwent a detailed history and physical examination that failed to identify any clinical evidence of hypopituitarism or anterior pituitary hormone hypersecretion. Additional work-up with biochemical testing included tests for morning cortisol, ACTH, free $\mathrm{T}_{4}, \mathrm{TSH}, \mathrm{LH}, \mathrm{FSH}$, total testosterone, prolactin and IGF-1. The results of these investigations were all within normal limits.

We also ordered a pituitary protocol MRI and confirmed the presence of a $2.1-\mathrm{cm}$ sellar lesion with suprasellar extension, causing a mild compressive effect on the optic chiasm. Imaging characteristics were consistent with features of an adenoma. Because of the lesion's compressive effect on the optic chiasm, the patient was referred to an ophthalmologist for formal visual field testing, which did not show any abnormalities.

After a patient-centred discussion of management options, the patient was referred to a neurosurgeon for consideration of surgical intervention. Surgery was subsequently arranged for two main reasons: radiographic evidence of compression on the optic chiasm and an increased risk of pituitary apoplexy relating to the patient's therapeutic anticoagulation treatment for atrial fibrillation. He underwent successful endoscopic transsphenoidal resection of his pituitary lesion several months later, with an uneventful postoperative course. The final pathology report showed that the lesion was consistent with a nonfunctioning pituitary macroadenoma.

\section{References}

1. Scangas GA, Laws ER Jr. Pituitary incidentalomas. Pituitary 2014;17:486-91.

2. Freda PU, Beckers AM, Katznelson L, et al.; Endocrine Society. Pituitary incidentaloma: an Endocrine Society clinical practice guideline. J Clin Endocrinol Metab 2011;96:894-904.

3. Galland F, Vantyghem MC, Cazabat L, et al. Management of nonfunctioning pituitary incidentaloma. Ann Endocrinol (Paris) 2015;76:191-200.

4. Sanno N, Ovama K, Tahara S, et al. A survey of pituitary incidentaloma in Japan. Eur J Endocrinol 2003;149:123-7. 
5. Teramoto A, Hirakawa K, Sanno N, et al. Incidental pituitary lesions in 1,000 unselected autopsy specimens. Radiology 1994;193:161-4.

6. Ezzat S, Asa SL, Couldwell WT, et al. The prevalence of pituitary adenomas. Cancer 2004;101:613-9.

7. Orija IB, Weil RJ, Hamrahian AH. Pituitary incidentaloma. Best Pract Res Clin Endocrinol Metab 2012;26:47-68.

8. Melmed S, Casanueva FF, Hoffman AR, et al.; Endocrine Society. Diagnosis and treatment of hyperprolactinemia: an Endocrine Society clinical practice guideline. J Clin Endocrinol Metab 2011;96:273-88.

9. Serri O, Chik CL, Ur E, et al. Diagnosis and management of hyperprolactinemia. CMAJ 2003;169:575-81.
Decisions is a series that focuses on practical evidence-based approaches to common presentations in primary care. The articles address key decisions that a clinician may encounter during initial assessment. The information presented can usually be covered in a typical primary care appointment. Articles should be no longer than 650 words, may include one box, figure or table and should begin with a very brief description ( 75 words or less) of the clinical situation. The decisions addressed should be presented in the form of questions. A box providing helpful resources for the patient or physician is encouraged.

\section{Competing interests: None declared.}

This article has been peer reviewed.

The clinical scenario is fictional.

Affiliations: Department of Medicine (Saeed), Division of Endocrinology and Metabolism,
University of Toronto, Toronto, Ont.; Department of Medicine (Braga), Division of Endocrinology and Metabolism, McMaster University, Hamilton, Ont.

Contributors: Both authors contributed equally to the development of this article, revised it critically for important intellectual content, gave final approval of the version to be published and agreed to be accountable for all aspects of the work .

Correspondence to: Omar Saeed, saeedo@ smh.ca 